\title{
Emergency surgical revascularisation for coronary angioplasty complications
}

John A Carey, Simon W Davies, Raphael Balcon, Clive Layton, Patrick Magee, Martin T Rothman, Adam D Timmis, John E Wright, Robin K Walesby

\begin{abstract}
Objectives-To evaluate trends in referrals for emergency operations after percutaneous transluminal coronary angioplasty (PTCA) complications; to analyse morbidity and mortality and assess the influence of PTCA backup on elective surgery.

Design-A retrospective analysis of patients requiring emergency surgical revascularisation within 24 hours of percutaneous transluminal coronary angioplasty.

Patients-Between January 1980 and December 1990, 75 patients requiring emergency surgery within 24 hours of percutaneous transluminal coronary angioplasty.

unit continues to require surgical cover. Delays in operating on stable patients in centres which operate a "next available theatre" backup policy may not differ from some units performing PTCA with offsite cover for PTCA complications. Particularly in the presence of multivessel disease, however, PTCA complications may be associated with the need for "crash" bypass and such patients are unlikely to survive hospital transfer. The proportion of patients requiring "crash" bypass has increased during the period reviewed because of the extent of disease in the emergency surgical group increased. These results indicate that surgery should not be denied to these patients.
\end{abstract}

Setting-A tertiary referral centre and postgraduate teaching hospital.

Results -57 patients (76\%) were men, the mean age was 55 (range 29-73) years, and $30(40 \%)$ had had a previous myocardial infarction. Before PTCA, 68 (91\%) had severe angina, $59(79 \%)$ had multivessel disease, and six (8\%) had a left ventricular ejection fraction of less than $40 \%$. A mean of $2 \cdot 1$ grafts (range one to five) were performed; the internal mammary artery was used in only one patient. The operative mortality was $9 \%$ and inhospital mortality was $17 \%$. There was a need for cardiac massage until bypass was established in 19 patients (25\%): this was the most important outcome determinant $(P$ $=0.0051$ ) and was more common in those patients with multivessel disease $(P=$ $0.0449)$ and in women $(P=0.0388)$. In 10 of the 19 cases a vacant operating theatre was unavailable, the operation being performed in the catheter laboratory or anaesthetic room. These 19 patients had an operative mortality of $32 \%$ and inhospital mortality of $47 \%$, compared with $2 \%$ and $7 \%$ respectively for the 56 patients who awaited the next available operating theatre. Complications included myocardial infarction, 19 patients (25\%); arrhythmias, 10 patients (3\%); and gross neurological event, two patients (3\%). The mean intensive care unit stay was 2.6 days (range 1 to 33 days) and the mean duration of hospital admission was 13 days (range 5-40 days).

Conclusions-Patients undergoing emergency surgery after PTCA complications have a substantially increased inhospital mortality and morbidity. PTCA in this

\section{(Br Heart f 1994;72:428-435)}

According to published work, emergency surgery after percutaneous transluminal coronary angioplasty (PTCA) is such a rare occurrence that, in selected patients, the procedure no longer warrants onsite surgical backup. Several centres have documented an extremely low incidence of emergency surgical procedures in the last few years. ${ }^{1-3}$ These data must be interpreted with extreme caution, as many variables affect angioplasty results. There are still many units which continue to require surgical intervention on a regular basis, particularly in specialised teaching institutions which perform many complex PTCA procedures; immediate surgical intervention may be lifesaving.

The inevitable expansion of angioplasty facilities, the continuing increase in procedures performed, and the widening indications for PTCA may have important implications for surgical units which provide emergency surgical cover, specifically if changing patient profiles have a bearing on operative results and if increasing numbers of emergency referrals have an adverse impact on elective surgery. Therefore, analysis of emergency surgical requirements for angioplasty complications must be performed accurately to plan logistical and financial resources in the future, to precisely define the implications of contractual cover for offsite angioplasty centres, and to ascertain the impact of changes in indications, equipment, or techniques on the incidence and outcome of emergency surgery.

The incidence of emergency surgery may 
vary between institutions (or vary in the same institution over time) because of differences in PTCA operator experience and aggressiveness, the angioplasty population, the availability of bail out techniques, and surgical referral and intervention criteria.

\section{Patients and methods}

This was a retrospective analysis of patient records. Information sources included anaesthetic and surgical records, angioplasty computer database, patient notes, and angiographic material. The inclusion criterion was surgery performed within 24 hours of failed angioplasty in the presence of clinical deterioration. Patients who had an operation within 24 hours of angioplasty for simple PTCA failure were excluded. Coronary lesions causing $50 \%$ stenosis or greater were considered as significant. Multivessel disease was defined as the presence of significant stenosis in more than one major coronary artery. Dyspnoea was graded according to the New York Heart Association classification; angina was graded by functional class as defined by the Canadian Cardiological Society. The incidence of PTCA related emergency surgical referrals was defined as the percentage of PTCA patients requiring emergency surgery. Intensive care occupancy was used for analysis of the impact of emergency surgery on waiting lists as the availability of intensive care unit beds is the limiting factor governing the number of operations that can be performed.

Percutaneous transluminal coronary angioplasty was offered to patients with cardiac pain unresponsive to medical treatment when the coronary anatomy was deemed suitable; patients who either declined angioplasty or were not considered suitable for it would invariably undergo surgical treatment unless the surgeons deemed them unsuitable. Percutaneous transluminal coronary angioplasty was not performed for anatomical reasons alone during diagnostic angiography.

Most PTCA procedures were performed by consultant or senior registrar grade operators; a few were performed by registrar grade operators in the presence of a senior colleague.

Between 1980 and 1983 full surgical standby was maintained during angioplasty procedures. This was then modified due to the rapid expansion of the angioplasty service and the decreasing incidence of emergencies. The presence of the surgical team on site was considered to be the minimum requirement and the next available operating theatre would be used if necessary.

Indications for surgical intervention included perforation of coronary vessel (with or without tamponade), intimal dissection with luminal narrowing unrelieved by further balloon inflations, immediate or delayed vessel occlusion due to intimal dissection, thrombosis, spasm unresponsive to vasodilators, and catheter tip detachment.

Bail out and support measures were restricted to inotropic pharmacological sup- port and, where necessary, closed or open cardiac massage. Perfusion balloons and catheters, intracoronary stents, and intra-aortic balloon counterpulsation were not used before surgery at this time.

Patients were subdivided into two groups on the basis of the degree of instability and need for immediate surgical revascularisation: group I, patients requiring "crash" bypass; and group II, patients requiring urgent bypass.

Group I included patients in asystole, intractable ventricular fibrillation, or profound shock unresponsive to inotropic support necessitating immediate cardiopulmonary bypass and surgical revascularisation. These patients required cardiac massage until bypass was established. Crash bypass was performed in the operating theatre if facilities were available at the time; otherwise operations were conducted in the catheter laboratory or anaesthetic room.

Group II patients showed instability but maintained systemic pressures (with or without inotropic drugs) which were considered compatible with adequate organ perfusion. Urgent surgical revascularisation was performed in the operating theatre as soon as facilities and staff were available.

Anaesthetic induction and maintenance were achieved using intravenous (benzodiazepine/opiate) drugs. All patients who had arrested were intubated and ventilated with $100 \%$ oxygen. In the remaining patients, particular attention was paid to pain control and the maintenance of steady mean arterial pressures and heart rate to avoid increases in myocardial oxygen demands. Most patients were given mannitol or a loop diuretic intraoperatively. A low dose dopamine infusion ( $<5 \mu \mathrm{g} / \mathrm{kg} / \mathrm{min}$ ) was started if impaired renal function had been identified preoperatively or in patients who did not have an adequate diuretic response during the course of the operation. In an effort to achieve some degree of additional cerebral protection, $2 \mathrm{~g}$ methylprednisolone or $20 \mathrm{mg}$ dexamethasone were administered to crash bypass patients. All patients were ventilated for at least four hours after the operation and received continuous intravenous nitrate infusions in the early postoperative period.

All patients had median sternotomy. Cardiopulmonary bypass was established after heparinisation $(3 \mathrm{mg} / \mathrm{kg})$, aortic and right atrial cannulation. In one patient who had undergone previous cardiac surgery, bypass was established using femoral cannulation. Intraoperative myocardial protection was provided by either anterograde crystalloid cardioplegia during aortic cross clamping, intermittent aortic cross clamping and ventricular fibrillation, or ventricular fibrillation without aortic cross clamping. The former two techniques are used routinely for elective surgery at this institution.

Patients were weaned from bypass on glyceryl trinitrate infusions. Inotropic support was instituted if systemic pressures were inadequate (systolic blood pressure $<90 \mathrm{mmHg}$ ) 


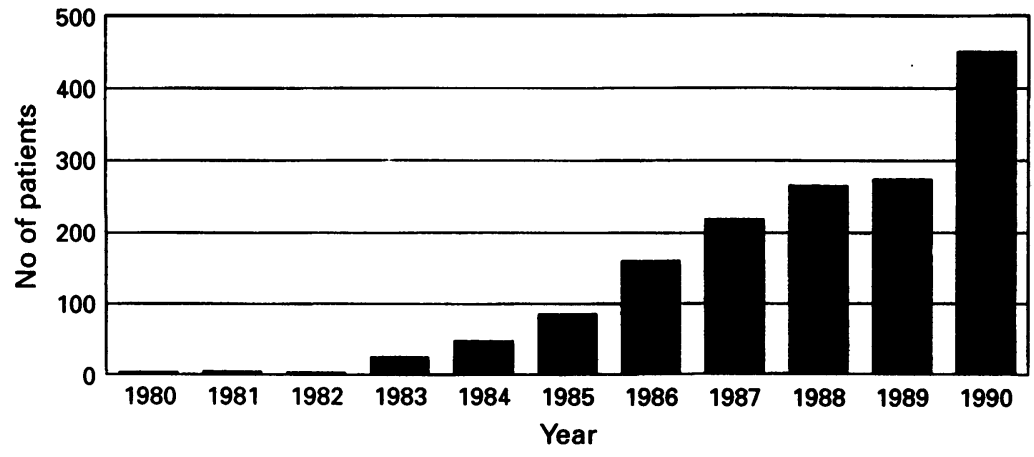

Figure 1 Rapid growth in angioplasty.

using adrenaline, dopamine, or dobutamime. The intra-aortic balloon was used after failure to wean from bypass on substantial inotropic support. Prophylactic insertion of the intraaortic balloon before weaning from bypass was occasionally used if ventricular damage was thought to be extensive when the heart was inspected after sternotomy.

Owing to the frequency of omission of creatine kinase MB enzymatic analysis, the difficulties in perioperative creatine kinase $\mathrm{MB}$ interpretation, and the frequency of non-specific transient $T$ wave changes, the total number of perioperative infarctions were determined as follows: $(a)$ in survivors, the diagnosis of perioperative myocardial infarction was made on the basis of the appearance of new pathological $Q$ waves with or without previous ST segment elevation; and $(b)$ in patients who died, on the basis of ST elevation greater than $2 \mathrm{~mm}$ in more than one topographically related lead.

Differences were analysed by $\chi^{2}$ significance

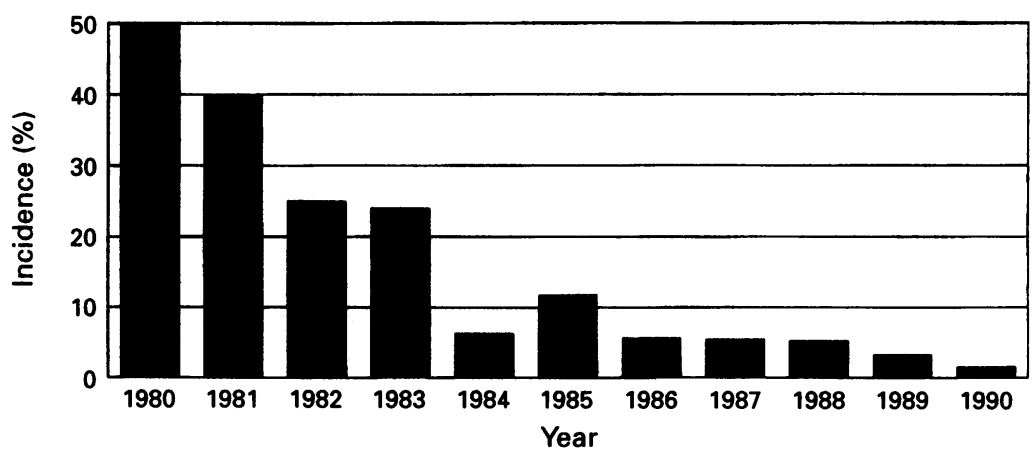

Figure 2 Decrease in incidence of emergency surgery after percutaneous transluminal coronary angioplasty.

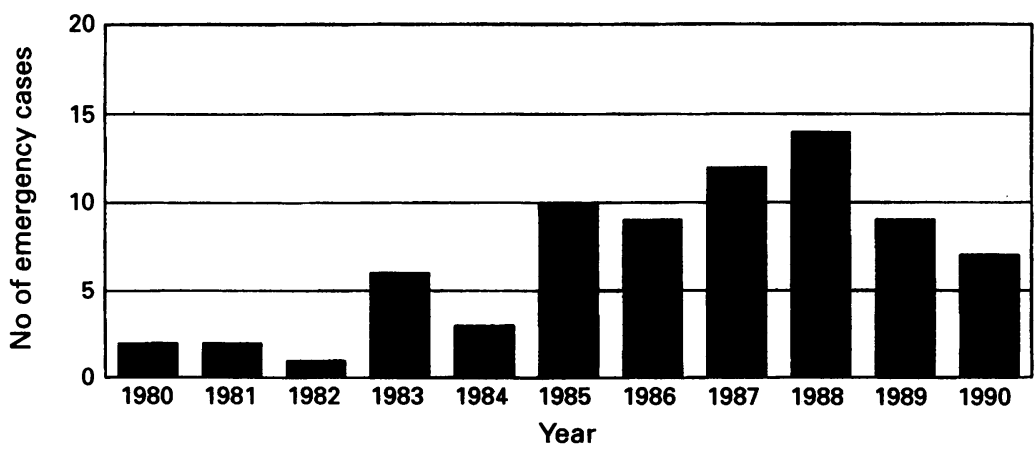

Figure 3 Increase in prevalence of emergency surgery after percutaneous transluminal coronary angioplasty. testing (categorical variables), Students's $t$ test (continuous variables, normal distribution), or the Mann-Whitney $U$ test (continuous variables, skewed distribution); forward stepwise logistic regression analyses were performed to determine the clinical, angiographic, procedural, haemodynamic, and surgical predictors of the need for crash bypass, operative and inhospital mortality: $P$ values of less than 0.05 were regarded as significant. Data are expressed as mean (SD) values unless stated otherwise.

\section{Results}

Angioplasty numbers have increased exponentially since 1980 and culminated in 450 angioplasty procedures during 1990 (fig 1). The sex ratio was $78.8 \%$ men and $21.2 \%$ women; the median age was 56.4 years (overall range $32-87$ years, mean age 56.7 years, semi-interquartile range $50 \cdot 1-63 \cdot 1$ years). Over the 11 year period, 75 of 1539 (4.9\%) patients required surgical revascularisation for clinical deterioration within 24 hours of failed angioplasty. Figure 2 illustrates the yearly incidence of emergency bypass after angioplasty complications expressed as a percentage of total procedures performed. The exponential increase in angioplasties performed was accompanied by a similar decrease in the incidence of emergency operations. Between 1986 and 1990, the mean incidence of emergency operations was $3 \cdot 7 \%$. Figure 3 shows the number of patients referred for an operation. The increase up to 1988 relates to the increase in the number of angioplasty procedures performed, with a $50 \%$ decrease in numbers referred in the last two years of the study despite a continuing increase in the number of PTCA procedures performed.

Table 1 summarises the characteristics of 75 patients undergoing an emergency operation after PTCA. Sixteen patients $(21 \%)$ had single vessel disease, 40 patients $(53 \%)$ had two vessel disease, and 19 patients $(25 \%)$ had three vessel disease. Before angioplasty, 11 patients $(15 \%)$ had stable angina, 22 patients $(29 \%)$ had recent onset of symptoms, 40 patients $(53 \%)$ had progressive angina, and two patients $(3 \%)$ had postmyocardial infarction pain. Forty five patients $(60 \%)$ had no

Table 1 Emergency surgical revascularisation with 24 hours of percutaneous transluminal coronary angioplasty (PTCA) failure: clinical profile in 75 patients. Values are mean (SD) or No (\%)

\begin{tabular}{lc}
\hline Patient profile before PTCA & \\
Age (years) & $55(9)$ (range 29-73) \\
No male & 57 \\
Previous myocardial infarction & $30(40)$ \\
Functional class & $7(9)$ \\
CCS class III & $68(91)$ \\
CCS class III/IV & $75(100)$ \\
Dyspnoea & $0(0)$ \\
NYHA grade O & $6(8)$ \\
NYHA grade I-IV & $64(85)$ \\
LVEF < 40\% & $2(1)$ (range 1-3) \\
Unstable angina & \\
No of vessels diseased &
\end{tabular}

CCS = Canadian Cardiovasacular Society; NYHA = New York Heart Association; $\mathrm{LVEF}=$ left ventricular ejection fraction. 


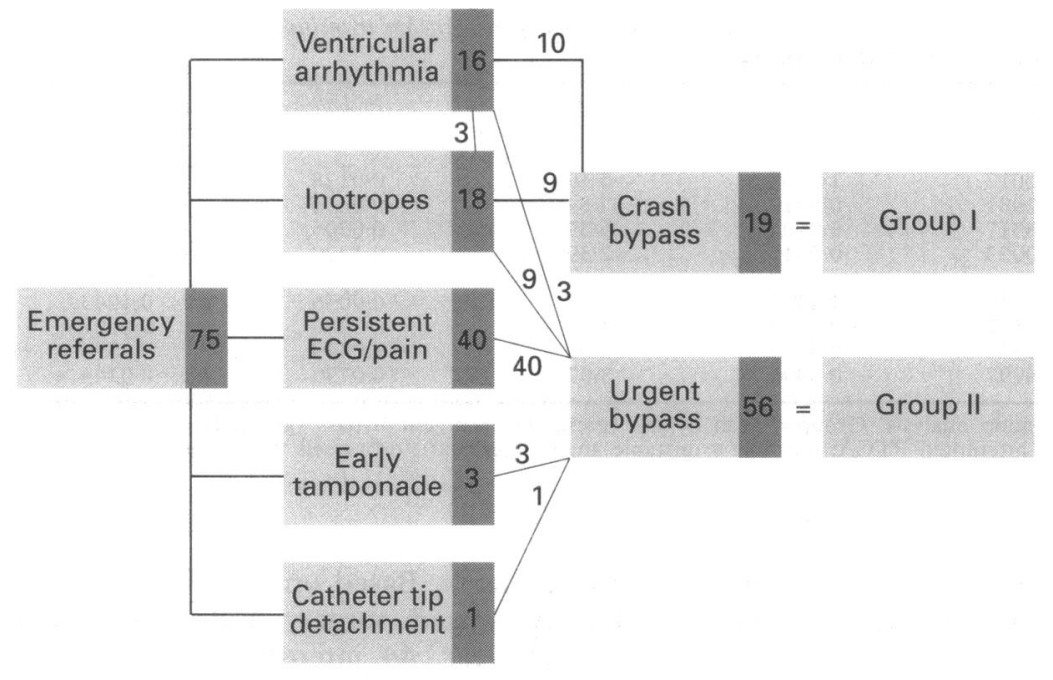

Figure 4 Preoperative patient status. patients, 99\%). The left internal mammary artery was harvested in two patients and used in one patient as a LAD bypass conduit. All significant coronary lesions were grafted and cardiopulmonary bypass was not reinstituted for further grafting.

Three methods of intraoperative myocardial protection were used: intermittent aortic cross clamp and ventricular fibrillation (57 patients, $76 \%$ ), anterograde, cold hyperkalaemic crystalloid cardioplegia administration and a single aortic cross clamp period (13 patients, $17 \%$ ), and ventricular fibrillation alone with no aortic cross clamp applied (five, $7 \%$ ). The mean number of bypass grafts performed was $2 \cdot 12$ (range 1-5). The mean cardiopulmonary bypass time was 64 (41) minutes and ranged from 22 to 150 minutes. This included 11 patients with prolonged bypass times ( $\geqslant 100$ minutes) and five patients in whom cardiopulmonary bypass was re-established to allow the heart a further period of rest as a non-working, beating ventricle, for inotropes to be increased, or an intra-aortic balloon to be inserted.

There were seven operative deaths $(9 \%)$. Of the five patients who required reinstitution of cardiopulmonary bypass after initial weaning, three patients could not be weaned successfully from bypass after further attempts despite increased inotropic and mechanical support. The intra-aortic balloon pump was also used before discontinuance in three patients. Of the eight patients with intra-aortic balloon pump support, two survived.

The mean number of ventilator hours was 34 (range 4-360 hours). The mean intensive care unit occupancy was $2 \cdot 6$ days (range $1-33$ days); 39 patients $(60 \%)$ were discharged to the ward on the first postoperative day, and a further 10 patients $(15 \%)$ on the second day.

Table 2 details the complications. Nineteen patients $(25 \%)$ had a myocardial infarction and this included the 13 deaths and six patients who were discharged alive from hospital with evidence of $Q$ wave infarction $(9 \%)$. Arrhythmias occurred in 10 patients (15\%), including atrial fibrillation in four patients $(6 \%)$, supraventricular tachycardia in one patient $(1 \%)$, and ventricular fibrillation in two patients (3\%); left bundle branch block, one patient ( $1 \%)$; right bundle branch block, two patients $(3 \%)$; and permanent pacemaker insertion, one patient (1\%).

Ultimately, six inhospital deaths followed in 68 operation survivors $(9 \%)$. These deaths Ten $(53 \%)$ patients underwent surgical revascularisation in the catheter laboratory or anaesthetic room.

Cardiopulmonary bypass was established after median sternotomy, aortic and right atrial cannulation in 73 patients. One patient who had previous coronary revascularisation was placed on cardiopulmonary bypass via the femoral route before sternotomy. One patient in persistent ventricular fibrillation underwent LAD (left anterior descending coronary artery) revascularisation without cardiopulmonary bypass (this patient was included in group II and survived).

Most patients were revascularised using reversed long saphenous vein alone (74
Table 2 Complications after emergency surgical revascularisation in 75 patients

\begin{tabular}{ll}
\hline & No (\%) patients $(n=75)$ \\
\hline Cardiac complications & \\
$\quad$ Myocardial infarction & $19(25)$ \\
Arrhythmias & $10(15)$ \\
Non-cardiac complications & $5(7)$ \\
Wound & $4(6)$ \\
Renal & $2(3)$ \\
Cerebral & $2(3)$ \\
Septicaemia & $7(9)$ \\
Mortality & $13(17)$ \\
$\quad$ Operative mortality & \\
Inhospital mortality & \\
\hline
\end{tabular}


Table 3 Determinants of hospital mortality and need for crash bypass after percutaneous transluminal coronary angioplasty (PTCA) related emergency surgical revascularisation

\begin{tabular}{|c|c|c|c|c|c|}
\hline Variable & $\beta$ estimate & Standard error & $\chi^{2}(\beta=0)$ & Probalitity $(\beta=0)$ & Last $R$ square \\
\hline \multicolumn{6}{|c|}{ Hospital mortality } \\
\hline Intercept & $-3 \cdot 2017$ & $1 \cdot 2868$ & $6 \cdot 19$ & 0.0128 & 0.0823 \\
\hline Group & $-2 \cdot 2931$ & 0.8912 & $7 \cdot 84$ & 0.0051 & 0.1020 \\
\hline Sex & 1.8317 & 0.7904 & $5 \cdot 37$ & 0.0205 & 0.0722 \\
\hline Stenosis (\%) & 0.0023 & 0.0015 & $2 \cdot 39$ & 0.1220 & 0.0335 \\
\hline \multicolumn{6}{|l|}{ Crash bypass } \\
\hline Intercept & $4 \cdot 0135$ & $1 \cdot 4478$ & $7 \cdot 68$ & 0.0056 & $0 \cdot 1043$ \\
\hline Sex & $-1 \cdot 3367$ & 0.6616 & $4 \cdot 27$ & 0.3880 & 0.0607 \\
\hline No vessels & -0.9536 & 0.4755 & 4.02 & 0.0449 & 0.0574 \\
\hline Unstable & 0.6997 & 0.3996 & 3.07 & 0.0799 & 0.0444 \\
\hline
\end{tabular}

Stepwise foreward logistic regression analysis. Group = crash bypass (group I) or urgent bypass (group II); stenosis (\%) = percentage stenosis of vessel for attempted PTCA; unstable = unstable angina (see text); no vessels = number ${ }^{\circ}$ of significantly diseased vessels (see text).

occurred at 1 (two patients), 2, 7, 14, and 54 days after the operation. Overall, the inhospital mortality rate was $17 \%$ (13 of 75 patients). The mean hospital stay in survivors was 13 days (range five to 40 days).

Patients who survived the operation had a longer intensive care and hospital stay than

Table 4 Comparison of preoperative, intraoperative, and postoperative variables in patients undergoing crash versus urgent bypass procedures. Values are mean (SD) or No $(\%)$

\begin{tabular}{|c|c|c|c|}
\hline & Crash bypass & Urgent bypass & Pvalue \\
\hline \multicolumn{4}{|l|}{ Pre-PTCA variables } \\
\hline Age (years) & $56.0(6 \cdot 35)$ & $54.95(9.27)$ & 0.6475 \\
\hline Male:female ratio & $12: 7$ & 45:11 & 0.1293 \\
\hline LVEF $<40 \%$ & $3 / 19(15 \cdot 8 \%)$ & $3 / 56(5 \cdot 4 \%)$ & 0.1475 \\
\hline Unstable angina & $16 / 19(84 \cdot 2 \%)$ & $48 / 56(85 \cdot 7 \%)$ & 0.2190 \\
\hline No of vessels diseased & $2.32(0.67)$ & $1.95(0.67)$ & 0.0419 \\
\hline Vessel stenosis (\%) & $88.22(10 \cdot 02)$ & $71 \cdot 22(188 \cdot 10)$ & 0.4254 \\
\hline Functional class (CCS) & $3.47(0.9)$ & $3.59(1.01)$ & 0.6586 \\
\hline \multicolumn{4}{|l|}{ Operative details } \\
\hline No of grafts & $2.44(0.70)$ & $1.96(0.83)$ & 0.0304 \\
\hline Initial CPB time & $105 \cdot 30(79 \cdot 55)$ & $55.27(21.90)$ & 0.0003 \\
\hline Total CPB time & $85.89(45 \cdot 12)$ & $58.69(33.03)$ & 0.0366 \\
\hline \multicolumn{4}{|l|}{ Results } \\
\hline Operative mortality & $6 / 19(32 \%)$ & $1 / 56(2 \%)$ & 0.0001 \\
\hline Ventilator hours & $34.64(36 \cdot 04)$ & $36 \cdot 54(80 \cdot 30)$ & $0 \cdot 2575$ \\
\hline ICU stay (days) & $2.23(1.79)$ & $2 \cdot 83(5 \cdot 45)$ & 0.7077 \\
\hline Hospital stay (days) & $8.06(8.04)$ & $11.57(8 \cdot 68)$ & $0 \cdot 1420$ \\
\hline Inhospital mortality & $9 / 19(47 \%)$ & $3 / 56(5 \%)$ & 0.0001 \\
\hline
\end{tabular}

LVEF = Left ventricular ejection fraction; $C B P=$ cardiopulmonary bypass time; $C C S=$ Canadian Cardiovascular Society; ICU = intensive care unit.

Table 5 Increased complexity of patients referred for emergency surgery after percutaneous transluminal coronary angioplasty (PTCA) failure in recent years. Values are mean (SD) or No (\%)

\begin{tabular}{llll}
\hline & Early years (1980-5) & Recent years (1986-90) & P value \\
\hline Pre-PTCA & & & \\
Age (years) & $53 \cdot 5(9 \cdot 6)$ & $56 \cdot 1(8 \cdot 1)$ & $0 \cdot 2646$ \\
Female sex & $3 / 24(12 \cdot 5 \%)$ & $15 / 51(29 \cdot 4 \%)$ & $0 \cdot 2120$ \\
Vessel disease & $1 \cdot 7(0 \cdot 75)$ & $2 \cdot 2(0 \cdot 6)$ & $0 \cdot 0034$ \\
Functional class (CCS) & $3 \cdot 3(1 \cdot 2)$ & $3 \cdot 6(1 \cdot 0)$ & $0 \cdot 2270$ \\
LVEF < 40\% & $3 / 24(12.5 \%)$ & $3 / 51(5 \cdot 9 \%)$ & $0 \cdot 3970$ \\
Unstable angina & $4 / 24(16 \cdot 7 \%)$ & $7 / 51(13.7 \%)$ & $0 \cdot 3252$ \\
Vessel stenosis (\%) & $88 \cdot 25(6 \cdot 88)$ & $88.34(8.66)$ & $0 \cdot 4594$ \\
\hline
\end{tabular}

CCS = Canadian Cardiological Society; LVEF = left ventricular ejection fraction.

Table 6 Increasing mortality associated with percutaneous transluminal coronary angioplasty (PTCA) failure necessitating emergency surgery in recent years. Values are No (\%) patients

\begin{tabular}{llc} 
& $\begin{array}{c}\text { Early years } \\
(1980-5)\end{array}$ & $\begin{array}{c}\text { Recent years } \\
(1986-90)\end{array}$ \\
\hline $\begin{array}{l}\text { Incidence of emergency surgery } \\
\text { PTCA patients for crash bypass }\end{array}$ & $24 / 171(14)$ & $51 / 1368(4)$ \\
$\begin{array}{c}\text { Crash bypass/total emergency patients } \\
\text { Operative status and surgical outcome } \\
\text { Crash bypass }\end{array}$ & $3 / 171(2)$ & $16 / 1368(1)$ \\
$\quad \begin{array}{l}\text { Operative mortality } \\
\text { Inhospital mortality }\end{array}$ & & $16 / 51(31)$ \\
Urgent bypass & $2 / 3(66)$ & \\
$\quad$ Operative mortality & $3 / 3(100)$ & $4 / 16(25)$ \\
Inhospital mortality & $0 / 21(0)$ & $6 / 16(38)$ \\
Overall results & $0 / 21(0)$ & $1 / 35(3)$ \\
Operative mortality & $2 / 24(8)$ & $3 / 35(9)$ \\
Inhospital mortality & $3 / 24(13)$ & $6 / 51(12)$ \\
\hline
\end{tabular}

elective patients. Based on a $3 \cdot 7 \%$ incidence of PTCA related surgery and 450 PTCA cases each year, 44 intensive care unit bed days were occupied by PTCA related emergency patients out of a total of 2200 available bed days, representing a $2 \%$ occupancy of the available intensive care unit beds. The 450 patients treated by PTCA during that year would have resulted in 433 fewer patients requiring an operation.

In relation to inhospital mortality, seven preoperative variables (age, sex, number of diseased vessels, presence of unstable angina, degree of vessel stenosis in PTCA vessel, left ventricular ejection fraction before PTCA, and group) were analysed by forward stepwise regression analysis: 20 iterations were performed and variables were removed if the $\chi^{2}$ value was less than $2 \cdot 0$. The two main determinants of inhospital mortality were patient group (crash versus urgent bypass, $P=$ $0.0051)$ and female gender $(P=0.0205)$. Apart from the degree of vessel stenosis before angioplasty $(P>0.05)$, none of the other variables had a $\chi^{2}$ value greater than $2 \cdot 0$ (table 3 ).

Table 4 documents the differences in preoperative, intraoperative, and postoperative variables between the two groups. Stepwise forward logistic regression analysis identified female sex and extent of vessel disease as the main determinants of the need for crash bypass (table 3 ).

Tables 5 and 6 give a comparison of the patient population, perioperative variables and outcome in those patients undergoing an emergency operation between early patients (from January 1980 to December 1985, 24 patients) and more recent patients (from January 1986 to December 1990, 51 patients).

\section{Discussion}

Information regarding PTCA related surgery in the United Kingdom is almost entirely based on outcome data collected from procedural registers from which detailed analyses are impossible. ${ }^{4}$ Two centres have published studies which are essentially descriptive. The first concluded that PTCA can be performed with offsite surgical cover in selected low risk (essentially single vessel disease) patients, with a $2 \cdot 3 \%$ incidence of emergency surgery and zero surgical mortality. ${ }^{1}$ The second concluded that patients with more extensive dis- 
ease ( 1.6 grafts per patient) had a zero operative mortality in the presence of onsite surgical facilities. ${ }^{2}$ These data do not reflect our experience to date, however, and give a false impression of surgical risk: many issues including changing trends in PTCA selection and in the patient population referred for emergency surgery, risk factors, and impact on elective surgery have not been addressed, issues which cannot be resolved by examination of data derived in other countries where patient profiles, number of patients, and surgical cover differ significantly. Within the United Kingdom itself, the incidence of emergency surgical revascularisation will vary widely between institutions for reasons other than patient selection for PTCA: experience and aggressiveness of angioplasty staff, availability of non-surgical bail out techniques, indications for surgical referral (or, even more importantly, non-referral), and availability or extent of surgical cover are equally relavent.

The safety of PTCA has improved dramatically with experience, even in the context of wider patient selection and more complex procedures. Two features distinguish the present series in that only one study has reported a greater extent of coronary artery disease in their emergency surgery population ${ }^{5}$ and only two studies have reported such a high degree of functional impairment. ${ }^{67}$ Mean age, the percentage female population, and incidence of previous myocardial infarction are typical. Unfortunately, complete patient profiles have not been reported in many papers.

We have shown that there is a continued need for onsite surgical cover in this experienced, high volume, angioplasty unit. Although emergency surgery is only required for a minority of patients undergoing PTCA, and the risk to individual patients has decreased, the numbers being referred for surgery are not decreasing to the same extent because of rapid PTCA expansion. The decrease in the number of surgical referrals in the final two years of the study are encouraging, but must be viewed cautiously. In recent years, almost one third of operations have been performed on collapsed patients who had no chance of survival without immediate surgical intervention. If these cases had occurred in PTCA units with offsite cover, these patients would not have survived hospital transfer. These data re-emphasise that first class management of patients undergoing PTCA mandates 24 hour, onsite surgical cover.

The requirement for emergency surgery after failed PTCA has been reported between $2 \%$ and $21 \% .{ }^{68}$ In general, recent reports quote a lower incidence than earlier studies. Neither our reported overall incidence of emergency surgery $(4.9 \%)$, nor the incidence in the last five years $(3.7 \%)$ are remarkable in the context of the periods covered. The status of patients referred for emergency surgery is an important feature of our study: $25 \%$ of emergency surgical candidates required cardiac massage to bypass. Even when patients developing "cardiogenic shock" were consid- ered in this category, only three other centres $^{9-11}$ have reported such a high incidence $(23 \%, 31 \%$, and $35 \%)$ and the remainder have documented a crash bypass/cardiogenic shock incidence between $1 \%$ and $16 \%$. As the reports do not document the number of moribund patients that did not undergo a salvage operation, the discrepancy in crash bypass rates may reflect differences in referral criteria. The extent of vessel disease was the major contributing factor to the high incidence of crash bypass in our series.

In respect of patients who require cardiac massage onto cardiopulmonary bypass, we would urge immediate transfer to the operating room where optimum operating conditions prevail. This series illustrates, however, that despite onsite surgical facilities and staff, it is not always possible to provide empty operating theatres for truly emergent cases due to routine surgical commitments. A total of $25 \%$ of these patients were referred at inopportune times during routine surgical operating sessions. We would advocate that surgical departments draw up specific contingency plans for such eventualities to ensure a rapid response capability.

Although the internal mammary artery is used in approximately $75 \%$ of elective coronary patients, the vessel was not used in $97 \%$ of these patients because of the additional ischaemic time involved in harvesting the artery and the risk of a low flow state postoperatively. Other centres have reported $0-24 \%$ internal mammary artery use after PTCA failure dependent on patient status ${ }^{12}{ }^{13}$; the true disadvantages of internal mammary artery harvesting after establishing cardiopulmonary bypass in this setting have yet to be clarified. The use of autoperfusion catheters has not resulted in increased internal mammary artery usage. " Published data pooled from a large number of USA centres reported an association between internal mammary artery usage and operative mortality in patients undergoing surgery for acute PTCA failure (internal mammary artery used, $1.03 \%$; no internal mammary artery used, $3 \cdot 24 \%$ ). The fact that no internal mammary artery was used in two thirds of the reported patients may, however, reflect a clinical factor not included in the data analysis which contributed to conduit selection: the percentage of patients in cardiogenic shock after PTCA was not reported (though internal mammary artery usage was associated with better mortality in the overall group undergoing surgery in the presence of cardiogenic shock); the number of patients who did not require $\mathrm{LAD}$ revascularisation was undefined; the use of autoperfusion catheters was not documented; and the relation between conduit selection and choice of intraoperative myocardial protection or the sequence of graft anastomoses was not explored. ${ }^{14}$

Of the 1539 patients who underwent PTCA, 1464 patients did not require surgery and, in this group, there was almost zero mortality. Indeed, the overall mortality for PTCA was less than $1 \%$ and compares with mortality 
figures for elective surgery. This study emphasizes, however, that the risk of emergency surgery after PTCA complications carries a high morbidity and mortality and that careful clinical judgement must be exercised in selecting patients for PTCA while awaiting the results of prospective randomised studies comparing medical treatment, angioplasty, and surgery.

The condition of the patient at the time of surgical referral is the primary outcome determinant. Even those patients who have waited for the next available operating room slot must be quoted risks in excess of routine elective coronary surgery. We have documented a $2 \%$ operative mortality and $5 \%$ inhospital mortality in this subgroup compared with about a $1 \%$ risk of inhospital mortality for routine surgery.

In comparison with previous reports, operative mortality (9\%) and hospital mortality $(17 \%)$ were high, but they again reflected patient status at the time of surgery and the extent of coronary disease. The difference in outcome after crash and urgent bypass was particularly remarkable. Operative mortality (32\%) was 16 times higher and hospital mortality (47\%) was seven times higher in the crash bypass group. From a different perspective, we believe that a $53 \%$ salvage rate in these circumstances is commendable and supports continuation of our present aggressive surgical policy. This should be compared with a $100 \%$ mortality reported in offsite referrals using the same intraoperative myocardial protection for patients in cardiogenic shock treated preoperatively with inotropes or intraaortic balloon counterpulsation and the inability to offer the opportunity for surgical salvage to patients who had intractable fibrillation or profound hypotension not responding to either of these two measures in the five referring centres. ${ }^{10}$

No prospectively randomised controlled trials comparing alternative techniques of intraoperative myocardial protection have been published to date. The disproportionate use of intermittent aortic cross clamping and ventricular fibrillation in our series precluded meaningful comparisons in this study. If surgical results are to be improved, particularly in the crash bypass group, improved perioperative myocardial protection and reperfusion strategies are imperative.

Initial cardiopulmonary bypass time was significantly longer in the crash bypass group due to the greater number of vessels bypassed and our impression that a further period of bypass with the heart in a beating but nonworking mode allows some degree of recovery of reversibly damaged muscle. Total bypass time was also significantly longer in the crash bypass group due to the more frequent need for the re-establishment of cardiopulmonary bypass as a consequence of poor left ventricular pump function.

In the light of the number of salvage cases, the incidence of gross neurological events is remarkably low (three of 19 patients, $16 \%$ ) and reflects effective resuscitation, good com- munication, and rapid institution of cardiopulmonary bypass (in the catheter laboratory itself if theatres are unavailable).

The incidence of periprocedural myocardial infarction by the appearance of $Q$ waves was undoubtedly underestimated in this study and reflected the retrospective nature of the study and diagnostic limitations. Quantification of troponin subunits should address this issue in the future.

Several workers have identified preprocedural risk factors for PTCA failure including multivessel disease, female sex, and lesion morphology..$^{15-18}$ These correlations serve as poor predictors of PTCA outcome. ${ }^{19}$ One group prospectively graded PTCA patients into low and high risk categories on the basis of accepted risk variables and provided full surgical backup for the latter group only. They failed to show any benefit from risk stratification as the incidence of emergency surgery was not significantly different between the two groups. ${ }^{20}$ Thus the requirement for emergency surgery after PTCA is unforeseeable.

Once this unpredictable event has occurred, what are the factors associated with poor outcome? We identified the key prognostic factors in the emergency surgery group specifically in relation to the need for crash bypass, operative and in hospital mortality. Extent of vessel disease and female sex were associated with an increased need for crash bypass which was the main outcome determinant. Age, severity of functional impairment (Canadian Cardiological Society class), left ventricular ejection fraction, unstable angina (pre-PTCA), infarction interval, or degree of vessel stenosis did not determine the need for crash bypass. Thus the status of the patient at the time of referral is the overwhelming outcome determinant and we can predict that the likelihood of crash bypass will increase as more complex PTCA procedures are performed in future years. This trend is already apparent in the present series. Comparing the results of emergency surgery in early patients (from January 1980 to December 1985, 24 patients) and more recent patients (from January 1986 to December 1990, 51 patients), the trend in actual numbers of patients referred did not mirror the dramatic decrease in the incidence of emergency surgical referrals ( $14 \%$ to $3.7 \%$ of PTCA patients). The patients referred for emergency surgery in the latter time period were characterised by an increase in the extent of vessel disease, which resulted in a higher proportion needing crash bypass and a poorer overall outcome. Autoperfusion balloons and non-surgical bail out (stenting and laser techniques) were not used in our unit during the period reported. Beyersdorf has reported an increase in the incidence of cardiogenic shock (currently $35 \%$ ) in surgical referrals in spite of the use of bail out techniques; he attributed this to the greater complexity of PTCA/stenting procedures being undertaken. ${ }^{10}$ Reports from other centres on bail out procedures are simply descriptive or contain important biases. 
Poststent treatment strategies (including surgical referral) were undertaken on the basis of individual discretion. Operator experience is a further confounding variable. Therefore there is insufficient data to support formulation of strict clinical policies or to evaluate the impact on emergency surgical referrals at this time..$^{21} 22$

The increase in PTCA related emergency surgery in the latter time period is a consequence of the rapid expansion of PTCA. Worldwide, the evolution of angioplasty has been phenomenally rapid. In the United Kingdom, the provision of angioplasty facilities and trained staff has fallen short of requirements. In contrast with the United States where the incidence of PTCA is 1000 cases per million population and PTCA cases outnumber coronary artery bypass procedures, only 100 cases per million population were performed in the UK in $1988 .{ }^{23}$ With the inevitable continuation in the expansion of PTCA, a fixed or increasing emergency rate will result in a greater impact on the limited resources provided for elective coronary artery bypass surgery. Notwithstanding the fact that 1464 patients with symptomatic coronary artery disease avoided the need for an operation, PTCA and surgery are complementary forms of treatment. Most patients awaiting elective surgery are unsuitable for PTCA and the number of surgical cases has not decreased despite the widespread use of PTCA. Emergency surgery results in the disruption of elective surgery and the use of intensive care beds. Surgical audit must evaluate the impact of emergency procedures (of which a variable proportion are PTCA related cases) on waiting lists. In this context, the current impact of PTCA related emergencies on elective surgery waiting lists is not insignificant, although outweighed by the avoidance of the need to join the surgical waiting list for many patients suitable for PTCA. Notwithstanding evidence that centres performing PTCA with offsite surgical cover have reported a lower incidence of emergency surgery $^{24}$ (and we do not know whether this reflects careful patient selection, more cautious operator practices, the absence of trainee operators, unwillingness or failure to publish poor results, or failure to provide the surgical option in moribund patients), surgical units providing contractual offsite cover should not underestimate the potential consequences. A prospective analysis of the impact of PTCA on elective surgical waiting lists, which would take into account present contractual arrangements for elective surgery, is warranted.

In conclusion, this study illustrates the continued need for 24 hour, onsite surgical cover for PTCA procedures in a high volume angioplasty unit. This need persists despite a dramatic decrease in the risk of an individual patient requiring surgical intervention for PTCA related complications. Expansion of PTCA and the increasing complexity of PTCA procedures have resulted in increased referral of patients needing immediate surgery which, on occassion, necessitates the institution of cardiopulmonary bypass in the catheter laboratory. Intensive surgical management is justified by a $53 \%$ hospital survival in the crash bypass group. If their complications had arisen in a PTCA centre without onsite surgical cover these patients are unlikely to have survived hospital transfer.

1 Richardson GS, Morton P, Murtagh JG, O'Keeffe BD, Murphy P, Scott M. Management of acute coronary occlusion during percutaneous transluminal coronary angioplasty: experience of complications in a hospital without on site facilities for cardiac surgery. $B M Y$ 1990;300:355-8.

2 Levy RD, Bennett DH, Brooks NH. Desirability of immediate surgical standby for coronary angioplasty. $\mathrm{Br} \mathrm{Heart}$ diate surgical stand

3 Shiu MF. Angioplasty: here's the proof [letter]. $\operatorname{Br} \mathcal{F}$ Hosp Med 1990;43:410-1.

4 Hubner PJ (British Cardiovascular Intervention Society). Cardiac interventional procedures in the United Kingdom in 1989. Br Heart $\mathcal{F} 1991 ; 66: 469-71$

5 Naunheim KS, Fiore AC, Fagan DC, et al. Emergency coronary artery bypass grafting for failed angioplasty: risk factors and outcome. Ann Thorac Surg 1989;47: 816-23.

6 Parsonnet V, Fisch D, Gielchinsky I, et al. Emergency operation after failed angioplasty. $\mathcal{f}$ Thorac Cardiovasc Surg 1988;96:198-203.

7 Stark KS, Satler LF, Krucoff MW, Rackley CE, Kent KM. Myocardial salvage after failed coronary angioplasty. $\mathscr{f}$ Am Coll Cardiol 1990;15:78-82.

8 Brahos GJ, Baker NH, Ewy G, et al. Aortocoronary bypass following unsuccessful PTCA: experience in 100 consecutive patients. Ann Thorac Surg 1985;40:7-10

9 Buffet P, Danchin N, Villemot JP, et al. Early and longterm outcome after emergency coronary artery bypas surgery after failed coronary angioplasty. Circulation 1991;84:III-254-9.

10 Beyersdorf F, Mitrev Z, Sarai K, et al. Changing patterns of patients undergoing emergency surgical revascularization for acute coronary occlusion. $¥$ Thorac Cardiovasc Surg 1993;106:137-48.

11 Borkon MA, Failing TL, Piehler JM, Killen DA, Hoskins ML Reed WA. Risk analysis of operative intervention for failed coronary angioplasty. Ann Thorac Surg 1992; 54:884-91.

12 Greene MA, Gray LA Jr, Slater AD, Ganzel BL, Mavroudis $C$. Emergency aortocoronary bypass after failed angioplasty. Ann Thorac Surg 1991;51:194-9.

13 Zapolanski A, Rosenblum J, Myler RK, et al. Emergency coronary artery bypass surgery following failed balloon angioplasty: role of the internal mammary artery graft. $f$ Cardic Surg 1991;6:439-48.

14 Edwards FH, Clark RE, Schwartz M. Impact of internal mammary artery conduits on operative mortality in coronary revascularization. Ann Thorac Surg 1994.57: 27-32.

15 Ellis SG, Vandormael MG, Cowley MJ, et al. Coronary morphologic and clinical determinants of procedural outcome with angioplasty for multivessel coronary disoutcome with angioplasty for multivessel coronary disease: implications

16 Ellis SG, Roubin GS, King SB, et al. In-hospital cardiac mortality after acute closure after coronary angioplasty: analysis of risk factors from 8,207 procedures. $\mathscr{\mathcal { ~ A m ~ C o l l }}$ Cardiol 1988;11:211-6.

17 Huber MS, Mooney JF, Madison J, Mooney MR. Use of morphological calcification to predict clinical outcome after dissection from coronary angioplasty. Am $¥$ Cardiol 1991;68:467-71.

18 Lincoff MA, Pompa JJ, Ellis SG, Hacker JA, Topol EJ Abrupt vessel closure complicating coronary angioplasty: clinical, angiographic and therapeutic profile. $\mathscr{f} \mathrm{Am}$ Coll Cardiol 1992;19:926-35.

19 Bergelson BA, Kyller MG, Jacobs AK, et al. Inadequacy of current criteria for predicting high risk coronary angioplasty [abstr]. ₹ Am Coll Cardiol 1990;15:206A

20 Iniguez A, Macaya C, Hernandez R, Alfonso F, Goicolea J, Casado J, Zarco P. Comparison of results of percutaneous coronary angioplasty with and without selective requirement of surgical standby. Am $\mathcal{f}$ Cardiol 1992 69:1161-5

21 Rothman MT, Davies SW. Intracoronary stents. Br Heart $\mathcal{f}$ 1992;67:425-7.

22 Leon MB, Wong SC. Intracoronary stents: a breakthrough technology or just another small step. Circulation 1994;89:1323-7.

23 British Cardiac Society Working Party. Coronary angioplasty in the United Kingdom. Br Heart $\mathcal{F} 1991 ; 66$ : plasty-32.

24 The Council of the British Cardiovascular Intervention Society. Surgical cover for percutaneous transluminal coronary angioplasty. $\mathrm{Br}$ Heart $\mathcal{F} 1992 ; 68: 339-41$. 